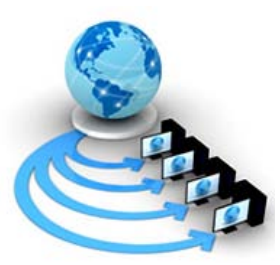

Volume 8, No. 9, November-December 2017

International Journal of Advanced Research in Computer Science

RESEARCH PAPER

Available Online at www.ijarcs.info

\title{
ANALYSIS OF RATING PREDICTION SYSTEM FROM TEXTUAL REVIEWS
}

\author{
M Sreenu \\ Assistant Professor \\ Dept.of Computer Science \& Engineering \\ SR Engineering College, \\ Warangal, Telangana, India
}

\author{
Sathu Anusha \\ M.Tech Scholar, \\ Dept.of Computer Science \& Engineering \\ SR Engineering College, \\ Warangal,Telangana,India
}

\begin{abstract}
In this work, we propose a slant based rating forecast technique to enhance expectation exactness in recommender frameworks. Right off the bat, it proposes a social client nostalgic estimation approach and computes every client's supposition on things. Furthermore, it considers a client's own wistful traits, as well as think about relational nostalgic impact. At that point, consider thing notoriety, which can be induced by the wistful conveyances of a client set that mirror clients' thorough assessment. Finally, by combining three variables client notion closeness, relational wistful impact, and thing's notoriety comparability into a recommender Framework to make an exact rating expectation. It leads an execution assessment of the three wistful components on a genuine informational index.
\end{abstract}

Keywords- Thing notoriety, Reviews, Rating expectation, Recommender Framework, Sentiment impact, User assumption.

\section{INTRODUCTION}

Because of its high notoriety, Web logs give an abundance of data that can be extremely useful in surveying the overall population's suppositions and assessments. It is in this way basic to investigate them and disinfect helpful learning that could be of financial esteems to sellers and other intrigued

parties.Whereas showcasing assumes an essential part for the recently discharged items, the general conclusion about the items may be vital to decide their achievement over the long haul. Investigating

the expansive volume of on-line audits accessible would deliver helpful noteworthy information that could be of financial esteems to merchants and other intrigued parties.Prediction of item execution is a to a

great degree space driven undertaking, for which a profound comprehension of an assortment of angles included is critical. Past reviews have affirmed that the suppositions communicated in the on-line audits are unequivocally connected with the business execution of items.

From the current reviews with respect to composing the reviews,on-line assessments, on-line remarks, exchange gatherings, the most stakes are taken by the film industry, which incorporates recordings, melodies, motion pictures, TV programs, and so forth. It is anything but difficult to get audits about motion pictures after or before its discharge from sites committed for films and it was hence chosen to take up motion picture as an item in this review. On the off chance that the expectation is centered around electronic merchandise, at that point it is required to consider diverse organizations/brands, yet here for motion pictures, it is conceivable to get correct measure of the movies income data. There has been past research and contrasting the outcomes and past outcomes was likewise another inspiration. Different monetary capacities have been used to inspect the connection between assessments found from item surveys and income development, stock exchanging volume change, and the offering value variety in business Websites, for example, e-Bay. Online networking is progressively being utilized by a huge segment of the populace in India. The substance created via web-based networking media sites have been to a great extent undiscovered by organizations for picking up client experiences and foreseeing genuine results. Microblogging administrations as of late have been a mainstream specialized device among Internet clients. It creates a large number of day by day messages for prominent sites. Microblogging is on-line informal marking like Twitter, is currently filling in as the electronic expression of mouth(eWOM), shaping an eWOM marking which depends on person to person communication and trust. Twitter has been overwhelmed with dynamic clients amid the most recent years and much consideration has been given in examining the social conduct and assessments of clients.

The concentrate of this review is on motion pictures as it is important to the online networking group. Substantial quantities of online networking clients talk about films and film industry incomes this information is effectively accessible from Internet Movie DataBase(IMDB). Microblogging destinations like Twitter additionally produce tweets which enable individuals to settle on choices about viewing a motion picture. The concentration of this exploration is film income forecast, however, can be reached out to other customer items.

In Fig.1, we instinctively demonstrate a case of positive audits and negative surveys on the site. From Fig.1, there are numerous positive words in a 5-star audit, for example, "great", and "stunning". Be that as it may, in a 2-star audit we discover negative words, such as "costly", and "poor". That implies a decent survey mirrors a high star-level and an awful audit mirrors a low-level.When we know the favorable circumstances and weaknesses from the two sorts of surveys, we can without much of a stretch settle on a choice. 

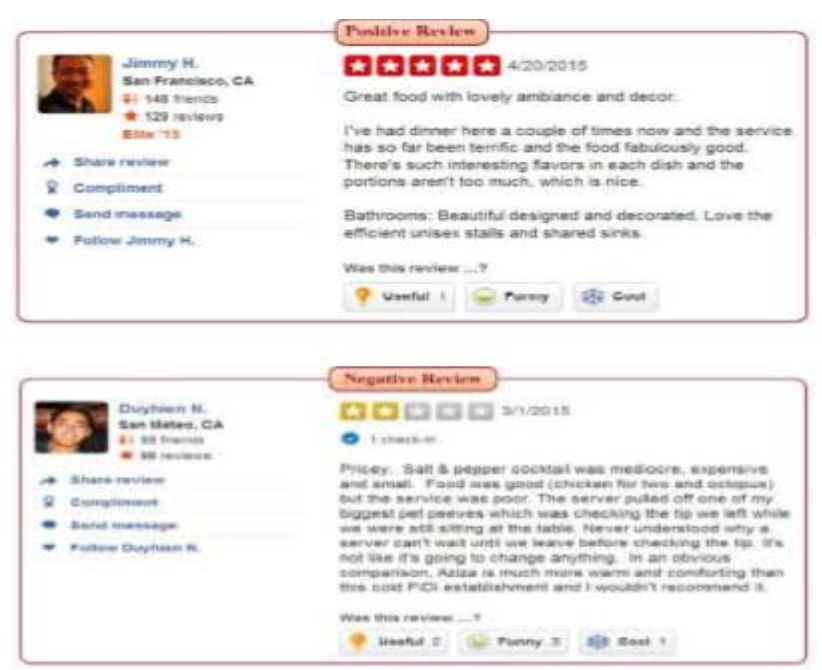

Fig. 1.An example of positive review and negative review on websites.

Regularly, if item"s audits reflect the positive opinion, at that point the thing might be with great notoriety. Oppositely, if thing's surveys are loaded with the negative notion, at that point the thing is in all probability with awful notoriety. So in view of clients audits notion, we can derive users" far - reaching evaluations on things.

In any case, it is troublesome for clients to settle on a decision when all applicant items reflect positive assumption or negative estimation. To settle on a buy a choice, clients not just need to know whether the item or thing is good, but likewise need to know how great the thing is. Is likewise concurred that distinctive individuals may have diverse wistful expression inclinations.

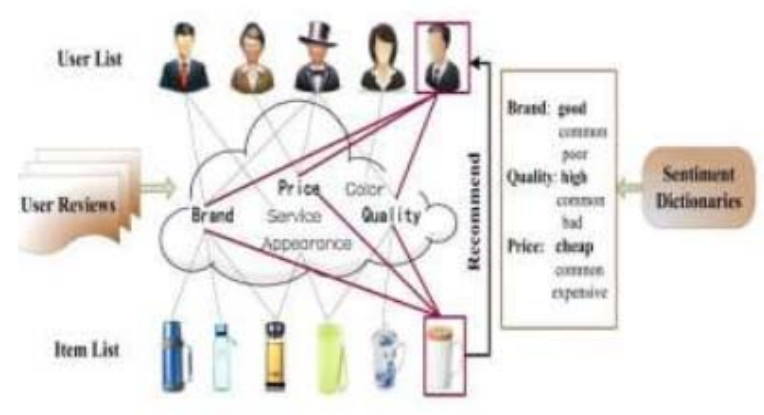

Fig. 2. The product features that user cares about are collected in the cloud including the words "Brand", "Price", and "Quality”, etc.

To address these problems,[12] propose a conclusion based rating expectation technique in the system of lattice factorization. In our work, we make utilization of social users" sentiment to gather appraisals. Fig. 2 is a case that shows our inspiration. In the first place, we separate item highlights from client audits. At that point, we discover the notion words, which are utilized to portray the item includes. Additionally, we use assumption word references to figure supposition of a particular client on a thing/item. In addition, we join social companion hover with estimation to prescribe. In Fig.2, the last client is keen on those item includes, so in view of the client audits and the conclusion word references, the last thing will be suggested. Contrasted (C) 2015-19, IJARCS All Rights Reserved and past work [6-8], the principle distinction is that: we utilize unstructured data to suggest rather than other organized social components. Contrasted and [3], [5], [9], the fundamental distinction is that: their work primarily concentrates on arranging clients into twofold assumption (i.e. positive or negative), and they don't go advance in mining user"s feeling. In our paper, we mine social user conclusion, as well as investigate relational nostalgic impact and item"s notoriety. At long last, we take every one of them into the recommender framework.

\section{BACKGROUND WORKS}

Lead a contextual investigation in the Movie Domain and Tackle the issue of digging audits for anticipating item deals execution. Examination indicates both feelings communicated in the audits and nature of the surveys have to affect on the future deal's execution of item. Conclusion PLSA (Probabilistic Latent Semantic Analysis)used for concealed feeling forecast from the archive. ARSA - Auto backward Sentiment-Aware Model for deals expectation. ARSQA - Auto backward Sentiment and Quality Aware Model to use both estimation and quality for anticipating item deals execution. For a Substantial volume of informational index Movie Review as a Case Study. Displaying conclusions in surveys can't be effortlessly tended to by regular content mining. An item deal is exceedingly area driven model. A Number of shrouded elements proposes a novel way to deal with supposition mining in light of Probabilistic Latent Semantic Analysis. SALSA concentrates on opinions instead of a point, it depends on record. Auto backward (AR) model is a period arrangement examination issue and particularly for monetary settings. Consolidating AR with assumption data mined from the audits. A New model for the item deals expectation called Auto backward Sentiment-Aware (ARSA) show for future deals execution. Organizations can have the capacity to better saddle the prescient energy of surveys and lead a business in a more powerful manner.

Shenghua Ba0, Shengliang Xu, Li Zhang, Rong Yan, Zhong Su, Dingyi Han, and Yong Yu (2012) acquainted work with distinguishing feelings in content [12][9][3][4]. This paper portrays tests worried with the planned examination of feelings in content. Expects to find and model the associations between on-line reports and client produced social feelings. Work select the related record in light of their passionate inclinations. It will use to foresee the feelings from the point of the report. The Goal is to precisely demonstrate the associations amongst words and feelings, and enhance the execution of its related errand, for example, feeling forecast. It won't discover the feeling in light of the substance of the report. Joint Emotion Topic Model by Latent Dirichlet Allocation, Emotion Term Model (Naïve-Bayes) and Emotion Topic Model (LDA) strategies are utilized. In this work, tests for associations between online records and client produced social emotions. Though the joint feeling point model is more adaptable and better ability it enhances the execution of social feeling forecast. In any case, it can't connect the associations between social feelings and full of feeling content. 
Alena Neviarouskaya, Helmut Prendinger, and MitsuruIshizuka (2011) which concentrated on the work for new notion vocabulary called SentiFul [1]. This paper depicts techniques to consequently produce and score another slant dictionary, called SentiFul and extend it through direct synonymy and antonymy relations, hyponym my relations, inference, and exacerbating with known lexical units. It expounded the calculation for automatic extraction of new assumption related mixes from Word Net [10] utilizing words from SentiFul as seeds for assessment conveying base parts and applying the examples of compound developments.

Inactive Semantic Analysis, PMI (Point astute Mutual Information) technique is utilized. SentiFul database that is contained are is risk vocabulary of supposition passing on terms, modifiers, useful words, and modular administrators which are essential for strong investigation of introduction, quality, and certainty level of the opinion reflected in the content. Finding new conclusion passing on words, especially through synonymy, antonymy, hyponymy relations, induction, and exacerbating strategies portrayed. As per the yielded comes about, the displayed approach can't make a difference in the genuine assignment of notion investigation.

Chenghua Lin, Yulan He, Richard Everson, Member, IEEE and Stefan Ruger (2012) concentrated on joint estimation subject model[5]. In this paper, novel probabilistic demonstrating structure called joint feeling subject (JST)model in light of inactive Dirichlet distribution (LDA)[12], which distinguishes estimation and theme all the while from content. Managed ways to deal with notion characterization which frequently neglects to deliver attractive execution when moving to different areas, the pitifully administered nature of JST makes it exceptionally convenient to different spaces. This is checked by the trial comes about on informational collections from five unique spaces where the JST demonstrate level out perform existing semi-directed methodologies in a portion of the informational collections notwithstanding utilizing no marked reports.

Feebly Supervised Learning, Joint Sentiment-Topic Model method is utilized. Joint slant theme demonstrates and a reparameterized variant of JST called Reverse-JST. Broad analyses led on informational collections crosswise over various areas uncover that these two models act distinctively when conclusion earlier learning is fused, in which case JST reliably out performe Reverse-JST. the JST demonstrate accomplished either better or equivalent execution contrasted with existing semi-administered approaches regardless of utilizing no marked archives. Be that as it may, the strategy not for new information and can't make a difference for the client's audit information.

\section{PROPOSED WORK}

The motivation behind the approach is to discover compelling intimations from surveys and anticipate social users" evaluations. We right off the bat separate item highlights from client survey corpus, and afterward we present the strategy for distinguishing sociäl users assessment. Finally, we intertwine every one of them into our estimation based rating expectation technique. It proposed a Highest rating suggestion framework for items and things. The commitments can be compressed as takes after:

1) It proposes a suggestion framework for sustenance items. To build up the proposal framework, rating datasets of items and things in the specific class which is utilized to peruse the printed surveys given by the clients.

The principle classifications which are utilized as a part of the application are only Lectures and books, Fashions, Food and Drink, Sports, Kids, and Family, Electronic machines. The datasets utilized as a part of this suggestion framework are "DouBan" and "Yelp" and other survey sites gives a wide idea in mining client inclinations and expectation user"s appraisals. Furthermore, ano ther dataset utilized is nothing but"On-line Product Rating" Dataset.

2) Textual surveys gotten from informational indexes is sorted into three sorts: To recognize positive audits, To distinguish negative surveys and To distinguish nonpartisan audits. With the assistance of the set sorts of surveys, we can distinguish the social connection between clients which will help to classifications the thing.

Fig 3 shows how review analysis is done form the original reviews on the websites.

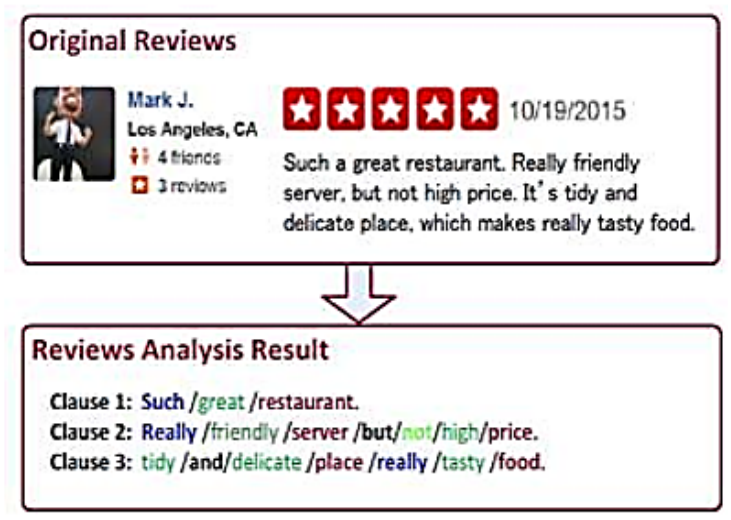

Fig. 3. An example of review analysis for identifying user" s sentiment

3) Sentimental lexicons will give the data of brands, quality, and cost on the premise of grid factorization.

This grid factorization can be performed by utilizing two sorts of strategies which are by applying conjunctive principles and another is by looking at item highlight and notion words.

4) This framework factorization technique will, at last, give the most astounding rating item proposal for a wide range of items and things to the client.

5) This proposal framework can be utilized by the client to choose which things to be requested or acquired and which are definitely not.

This proposal framework will take any choices for an item gathering. 


\section{CONCLUSION}

In this paper, a proposal model is recommended by mining supposition data from social useïssurveys. We propose social user"s conclusion estimation philosophies in light of the mine feeling words and assumption degree words from users" audits. We rage client conclusion comparability, relational estimation impact, and thing notoriety closeness into a bound together grid factorization structure to achieve the rating expectation undertaking. This rating item proposal framework which can be additionally utilized as the social connection coordinated effort demonstrates which can be utilized to perceive the social connection between the clients. The legit surveys will give the rating expectation simple and client will effectively get the result in the favored time.

\section{REFERENCES}

[1] Xiaohui Yu, Member, IEEE, Yang Liu, Member,IEEE, Jimmy Xiangji Huang, Member, IEEE, and Arjun A, Member, IEEE, "Digging On-line Reviews for Predicting Sales Performance: A Case Study in the Movie Domain" IEEE Transactions on Knowledge and Data Engineering, (Volume: 24, No: 4) Page(s): 720- 734, 2012.

[2] AlenaNeviarouskaya, Helmut Prendinger, andMitsuru Ishizuka, Member, IEEE, "SentiFul: A Lexicon for Sentiment Analysis" IEEE Transactions on Knowledge and Data Engineering, IEEE Transactions On Affective Computing Volume: 2, No: 1 pp: 22-36, 2011.

[3] Alexandre Trilla and FrancescAlías, Member, IEEE, "Sentence-Based Sentiment Analysis forExpressive Textto-Speech" IEEE Transactions On Audio, Speech, And Language Processing, Volume: 21, No: 2 pp: 223 - 233, 2013.
[4] Adam S, Stan Szpakowicz, 'Recognizing feeling Expressions from Text', Springer-Verlag, BerlinHeidelberg 2007.

[5] AnindyaGhose and Panagiotis G.Ipeirotis," Estimating the Helpfulness and Economic Impact of Product Reviews: Mining Text and Reviewer Characteristics", IEEE TRANSACTIONS ON KNOWLEDGE AND DATA ENGINEERING, VOL. 23, NO. 10,2011.

[6] Cecilia OvesdotterAlm., Dan Roth., RitchardSproat., 'Feelings from Text: Machine Learning for Text Based Emotion Prediction', ACL, pp.579-586, 2006.

[7] Chenghua Lin, Yulan He, Richard Everson, Member, IEEE, and Stefan Ruger, "Feebly Supervised Joint SentimentTopic Detection from Text" IEEE Transactions on Knowledge and Data Engineering, Volume: 24, pp: 1134 -1145, 2012.

[8] Jianping Cao, KeZeng, Hui Wang, Member,IEEE, Jiajun Cheng, FengcaiQiao, Ding Wen,Senior Member, IEEE, and YanqingGao, Member, IEEE, "Online Traffic Sentiment Analysis: Methods and Applications" IEEE Transactions On Intelligent Transportation Systems, Volume: 15, No: 2 pp: 844 - 853,2014.

[9] Minqing $\mathrm{Hu}$ and Liu $\mathrm{B}$, "Mining and Summarizing Customer Reviews," Proc. 10thACM SIGKDD Int'l Conf. Information Discovery and Data Mining SIGKDD '04, pp.168-177, 2004.

[10] Mouthmi. K, Nirmala Devi K., MuraliBhaskaranDr V, "Estimation Analysis and Classification Based on Textual Reviews", IEEE Conference, 2013.

[11] T. Nakagawa, K. Inui, and S. Kurohashi, "Reliance treebased estimation order utilizing CRFs with Hidden Variables," NAACP, 2010,pp.786-794.

[12] Xiaojiang Lei, XuemingQian, Member, IEEE, and Guoshuai Zhao,"Rating Prediction in light of Social Sentiment from Textual Reviews," IEEE Transactions On Multimedia, MANUSCRIPT ID: MM-006446. 